\title{
Antimicrobial Resistance in Salmonella enterica Serovar Paratyphi B Variant Java in Poultry from Europe and Latin America
}

\author{
L. Ricardo Castellanos, ${ }^{1}$ Linda van der Graaf-van Bloois, Pilar Donado-Godoy, \\ Kees Veldman, Francisco Duarte, María T. Acuña, Claudia Jarquín, François-Xavier Weill, \\ Dik J. Mevius, Jaap A. Wagenaar, Joost Hordijk, ${ }^{2}$ Aldert L. Zomer ${ }^{2}$
}

\begin{abstract}
Salmonella enterica serovar Paratyphi B variant Java sequence type 28 is prevalent in poultry and poultry meat. We investigated the evolutionary relatedness between sequence type 28 strains from Europe and Latin America using time-resolved phylogeny and principal component analysis. We sequenced isolates from Colombia, Guatemala, Costa Rica, and the Netherlands and complemented them with publicly available genomes from Europe, Africa, and the Middle East. Phylogenetic time trees and effective population sizes $\left(N_{e}\right)$ showed separate clustering of strains from Latin America and Europe. The separation is estimated to have occurred during the 1980s. $N_{e}$ of strains increased sharply in Europe around 1995 and in Latin America around 2005. Principal component analysis on noncore genes showed a clear distinction between strains from Europe and Latin America, whereas the plasmid gene content was similar. Regardless of the evolutionary separation, similar features of resistance to $\beta$-lactams and quinolones/fluoroquinolones indicated parallel evolution of antimicrobial resistance in both regions.
\end{abstract}

The d-Tartrate fermenting, nonparatyphoidal vari1 ant of Salmonella enterica serovar Paratyphi B, contemporarily known as variant Java, was first reported in 1935 by De Moor (1). From then until recently, Salmonella

\footnotetext{
Author affiliations: Utrecht University, Utrecht, the Netherlands

(L.R. Castellanos, L. van der Graaf-van Bloois, D.J. Mevius,

J.A. Wagenaar, J. Hordijk, A.L. Zomer); Corporación Colombiana de Investigación Agropecuaria-AGROSAVIA, Cundinamarca, Colombia (P. Donado-Godoy); Wageningen Bioveterinary Research, Lelystad, the Netherlands (K. Veldman, D.J. Mevius, J.A. Wagenaar); Instituto Costarricense de Investigación y Enseñanza en Nutrición y Salud, Tres Ríos, Costa Rica (F. Duarte, M.T. Acuña); Universidad del Valle de Guatemala, Guatemala City, Guatemala (C. Jarquín); Institut Pasteur, Paris, France (F.-X. Weill)
}

DOI: https://doi.org/10.3201/eid2606.191121
Paratyphi B var. Java caused sporadic self-limiting gastrointestinal infections in humans (2-4). Since 1990, reports of human infections increased in Europe (5-10), North America (11-15), and Australia (16,17). Human infections with this serovar were mainly related to exposure to fish or reptiles (16-18). A dramatic increase in prevalence in poultry and poultry meat in Europe was reported $(19,20)$, accompanied by an increase in antimicrobial resistance of the strains $(5,19)$.

Using a multilocus sequence typing (MLST) scheme for S. enterica (21), strains of Salmonella Paratyphi B var. Java were classified into different sequence types (STs). In a previous study, the use of both traditional serotyping and MLST was instrumental in identifying reptiles as the main source of Salmonella Paratyphi B var. Java infections in humans in Germany. Isolates originating from poultry were strongly associated with ST28, those from reptiles with ST88, and those from humans mainly with ST43 and ST149 (18). Connor et al. confirmed association of Salmonella Paratyphi B var. Java ST28 with samples originating from poultry with whole-genome sequence (WGS) data (22). Isolates of Salmonella Paratyphi B var. Java from poultry and food products have been identified as carriers of $\mathrm{mcr}$ gene variants conferring resistance to colistin $(23,24)$ and class 1 integrons in conjugative plasmids (5). Furthermore, near-identical plasmids carrying genes conferring resistance to third-generation cephalosporins were identified in Escherichia coli, Salmonella Paratyphi B var. Java ST28, and other Salmonella serovars known to cause infections in humans, such as Heidelberg and Enteritidis (25). In these regards, poultry-associated ST28 is of public health concern because it can be a reservoir of antimicrobial

${ }^{1}$ Current affiliate: Quadram Institute Bioscience, Norwich, UK.

${ }^{2}$ These authors contributed equally to this article. 
resistance to other Salmonella serovars of relevance to humans and species of Enterobacteriaceae in poultry and food products.

Previous reports from Latin America countries have identified Salmonella Paratyphi B var. Java as highly prevalent in poultry and poultry meat (26-30). Comparisons between strains of Salmonella Paratyphi B var. Java ST28 from Colombia and Europe showed a phylogenetic separation between isolates from Colombia and Europe (31). We then hypothesized that the separate lineage of Colombia strains could be part of a larger lineage of Salmonella Paratyphi B var. Java ST28 circulating in Latin America (31). Further investigation of this hypothesis could help identify potential events in poultry management (e.g., farming and trade) leading to the emergence and successful spread of Salmonella Paratyphi B var. Java ST28 in both regions. Our objective was to compare the evolutionary and antimicrobial resistance relatedness of poultry-associated Salmonella Paratyphi B var. Java ST28 from Europe and Latin America using WGSbased phylogenetic and temporal analysis.

\section{Methods}

\section{Strain and Genome Collection}

Our study comprised isolates from countries in Latin America and Europe that were previously serotyped as Salmonella Paratyphi B var. Java. We selected strains from different countries as follows.

\section{Colombia}

We used 259 epidemiologically independent isolates from broilers and broiler meat. We obtained isolates from baseline studies in poultry conducted in Colombia during 2008-2013 that originated from 25 samples from farms, 49 samples from slaughterhouses, and 185 samples from retail meat (32). As a rule of thumb, $20 \%$ of these isolates were randomly sampled using the select cases option in SPSS Statistics 24 (IBM, https:/ /www.ibm.com). As a result, we selected 52 isolates, 5 originating from samples from farms, 5 from samples from slaughterhouses, and 42 from samples from retail.

\section{Costa Rica}

We selected all available strains from a previous study to determine the prevalence of Salmonella spp. in chickens at slaughter in the country in 2009 (33). The Costa Rican Institute for Research and Training in Nutrition and Health (Tres Ríos, Costa Rica) made 15 isolates available and provided 3 nonbroiler strains of human, reptile, and swine origin.

\section{Guatemala}

Available strains from a previous study to determine the prevalence of Salmonella in retail raw chicken carcasses in the country were included (29). The Center for Health Studies from the Universidad del Valle de Guatemala (Guatemala City, Guatemala) made 5 isolates available.

\section{The Netherlands}

Isolates were collected in the Netherlands as part of the Monitoring of Antimicrobial Resistance and Antibiotic Usages in Animals program (34). Our study comprised 1,279 isolates obtained during 2000-2016. The isolates originated from broilers, broiler meat, and chicken products (1,100 isolates), human enteric infections (159 isolates), and other animals and food items (20 isolates). A stratified random sampling was performed with the Transform, Rank and Select Cases options in SPSS Statistics 24 for every year within the samples from broilers and broiler meat. In addition, we included 2 randomly selected isolates from the entire pool of human isolates and 2 from other animals and food items as nonbroiler references. As a result, 21 isolates originating from 16 broilers and 1 broiler meat sample, 2 human enteric infections, and 2 from other animals and food products were selected.

\section{Historical Salmonella Paratyphi B Var. Java ST28 Strains}

Two strains of human origin from Saudi Arabia isolated in 1987 (IP_6155/87) and 1992 (IP_7734/92) and 1 strain from a turkey of Israel origin isolated in Austria in 1988 (IP_6395/88) were identified from their MLST profiles. These strains were the earliest Salmonella Paratyphi B var. Java ST28 strains from the EnteroBase database (https://enterobase.warwick. ac.uk) (35).

\section{Publicly Available WGS Sequences}

We queried EnteroBase for assembled genomes using the "experimental data" search option for strains belonging to "ST28" in the "Achtman 7-gene MLST" scheme (accessed February 22, 2018). We selected 65 strains with metadata available for year and country of isolation for phylogenetic and temporal analysis (Appendix 1, https://wwwnc.cdc.gov/EID/ article/26/6/19-1121-App1.xlsx).

\section{WGS and In Silico Screening of Antimicrobial Resistance Genes and Plasmid Content}

We isolated genomic DNA from selected strains from the Netherlands, Colombia, Costa Rica, and Guatemala using the UltraClean Microbial DNA Isolation Kit (QIAGEN, https://www.qiagen.com). We performed 
WGS on the MiSeq platform using $2 \times 250$-bp reads and the NextSeq platform using two $2 \times 150$-bp reads (Illumina, https://www.illumina.com). WGS of historical strains was performed at the Plateforme de microbiologie mutualisée from the Pasteur International Bioresources network (PIBnet, Institut Pasteur, https://www.pasteur.fr) as previously described (36). We assembled the genomes of historical and newly sequenced strains with SPAdes version 3.10.1 (37) and screened for antimicrobial resistance genes and chromosomal mutations using ResFinder 3.1 (38). We subtyped plasmids using PlasmidFinder 2.0 and plasmid MLST (pMLST) 2.0 (39). For newly sequenced genomes, we performed 7-gene MLST at the strain level with MLST 1.8 (40).

\section{Phylogenetic Time Trees and Effective Population Size Estimates}

For phylogenetic single-nucleotide polymorphism (SNP) analysis of the core genome, we aligned WGS of all Salmonella Paratyphi B. var. Java ST28 isolates using Parsnp version 1.2 (41), excluding non-ST28 strains. We used Gubbins (42) to detect and visualize recombination regions in the core genome alignment and performed time-resolved phylogeny on recombination-filtered SNPs of the Salmonella Paratyphi B. var. Java ST28 isolates that were extracted from the Gubbins results and used for divergence dating in BEAST (43). We included only newly obtained genomes with coverage $>30$. We used isolation dates as tip dates in the phylogenetic tree, as outlined in the BEAST manual, with the following modifications: 10,000,000x sampling and a general time-reversible model plus $\gamma$ correction as the distance model. We tested a strict clock, relaxed logarithmic clock, and relaxed exponential clock as the clock model. We used a Bayesian skyline plot with 3 groups as demographic models to adjust for expected population changes and the effective sample size (ESS) method to select the strict clock model because it had the highest ESS values. All ESS values obtained with the strict clock model were $>1,000$. To generate Bayesian skyline plots for the European and Latin American populations, we repeated time-resolved phylogeny analysis using BEAST on 2 subsets containing the historical isolates and the isolates from Europe and Latin America.

\section{Orthology Prediction and Plasmid/Chromosome Contig Scoring}

We annotated genomes using Prokka version 1.13 (44), followed by orthology predictions using Roary (45). We differentiated chromosome and plasmid contigs with an in-house built tool. In brief, we scored contigs for the presence of known plasmid genes (https://github.com/aldertzomer/RFPlasmid), single-copy chromosomal marker genes (46), and kmer profiles and inferred their likely origin (plasmid or chromosomal) using a Random Forest model trained on known plasmid and chromosome assemblies (A.L. Zomer, unpub. data).

\section{Comparison of Accessory Genome}

We conducted principal component analysis (PCA) for all isolates on the gene presence/absence tables from the output of orthology predictions. We made comparisons using the accessory (noncore) genome of chromosome contigs, complete plasmid composition with all plasmid contigs, and plasmid composition with only plasmid contigs $\geq 50 \mathrm{~kb}$. We made additional characterizations of prophage sequences using the PHAge Search Tool Enhanced Release (47) and BLAST (https://blast.ncbi.nlm. nih.gov/Blast.cgi).

\section{Data Availability}

We deposited sequences of the historical and newly sequenced strains in the short-read archive of the European Nucleotide Archive (ENA) under project no. PRJEB31547. Accession numbers of all collected genomes are provided (Appendix 1 Table 1).

\section{Results}

\section{Phylogenetic Time Tree and Effective Population Size of Salmonella Paratyphi B var. Java ST28}

Most of the strains from poultry that we sequenced from Colombia (48/52), Costa Rica (15/15), Guatemala (4/5), and the Netherlands (17/17) belonged to ST28 (Table). Similarly, nonbroiler strains from a fish product and a turkey in the Netherlands and 1 from a pig carcass in Costa Rica belonged to ST28. Isolates from 3 human and 1 reptile samples from Costa Rica or the Netherlands belonged to STs different from ST28 (Appendix 1 Table 1).

In the phylogenetic trees, the strains collected from the Latin America countries formed a separate cluster. In contrast, the strains collected from the Netherlands clustered with strains originating from other countries in Europe (Figure 1). An additional cluster was formed by the historical strains, which were neither from Europe nor Latin America. The molecular clock was estimated at $3.5 \times 10^{-7}$ substitutions/site/year (1.7 SNPs/genome/year, 95\% CI 1.44-2.0 SNPs/genome/year). The output of BEAST indicated separation between strains from Europe and Latin America occurred in 1987 
Antimicrobial Resistance in Salmonella Paratyphi B

Table. Newly obtained and publicly available genomes of 155 Salmonella enterica serovar Paratyphi B variant Java sequence type 28

\begin{tabular}{|c|c|c|c|}
\hline Source & Total & No. per source* & Years isolated \\
\hline \multicolumn{4}{|l|}{ Historical } \\
\hline Saudi Arabia† & 2 & 2 human & 1987-1992 \\
\hline Austria† & 1 & 1 poultry $\ddagger$ & 1988 \\
\hline \multicolumn{4}{|l|}{ Europe } \\
\hline Belgium§ & 5 & 5 unknown & 2014 \\
\hline Denmark§ & 9 & 8 poultry, 1 unknown & 2009-2015 \\
\hline Germany§ & 5 & 3 poultry, 1 human, 1 unknown & $2001-2013$ \\
\hline Ireland§ & 2 & 2 human & $2015-2016$ \\
\hline Nigeria§̧ & 1 & 1 poultry & 2009 \\
\hline The Netherlands $\dagger$ & 19 & 18 poultry, 1 fish & $2000-2016$ \\
\hline United Kingdom§ & 24 & 18 unknown, 5 human, 1 bovine & 2006-2017 \\
\hline \multicolumn{4}{|l|}{ Latin America } \\
\hline Colombia†\# & 67 & 67 poultry & 2008-2013 \\
\hline Costa Rica† & 16 & 15 poultry, 1 swine & 2009-2014 \\
\hline Guatemala† & 4 & 4 poultry & 2012 \\
\hline \multicolumn{4}{|c|}{$\begin{array}{l}\text { *Source-metadata of publicly available genomes was obtained from EnteroBase (https://enterobase.warwick.ac.uk). } \\
\text { †Newly obtained. } \\
\text { †Sample from a turkey imported from Israel. } \\
\text { §Publicly available. } \\
\text { TPhylogenetically related to the European clade. } \\
\text { \#19 genomes from previous reports in Colombia were publicly available in EnteroBase (31). }\end{array}$} \\
\hline
\end{tabular}

(95\% CI 1978-1988) (Figure 1; Appendix 2 Figure 1, https://wwwnc.cdc.gov/EID/article/26/6/191121-App1.pdf). From the Bayesian Skyline plot, we inferred that the effective population size $\left(N_{e}\right)$ of strains from Europe increased sharply in 1995 (95\% CI 1992-1998) and in Latin America in 2005 (95\% CI 2001-2007) (Figure 2), 10 years later than in Europe.

\section{In Silico Characterization of Frequent Antimicrobial Resistance Genes and Plasmid Subtypes}

We found a chromosomal class 2 integron (with dfrA1sat1-aadA1 [GenBank accession no. AB188271.1]) in historical strain IP_6155/87, collected in 1987 in Saudi Arabia. However, this integron was not in the other 2 historical strains from Austria (originating from Israel [IP_6395/88]) and Saudi Arabia [IP_7734/92]). In strain IP_6155/87, the alignment of the class 2 integron was divided into multiple contigs. We also found the integron in all strains from Europe and Latin America in contigs of $\approx 50 \mathrm{~kb}$ carrying the complete integron or divided into multiple contigs. Alignments of $\approx 50 \mathrm{~kb}$ contigs carrying the complete integron, revealed $100 \%$ identity within and between strains from European and Latin American clades.

Resistance to $\beta$-lactams in strains from Europe was mainly mediated by $b l a_{\mathrm{TEM}-1 \mathrm{~B}}$ or $b l a_{\mathrm{TEM}-1 \mathrm{~B}}$-like genes. These genes were found in $64 \%$ of isolates in the European clade. In strains from the Latin American clade, $b l a_{\mathrm{CMY}-2}$ was most prevalent and was carried in $50 \%$ of strains (Figure 1). bla $a_{\text {ТЕМ-1в }}$ gene is known to confer resistance to aminopenicillins and $b l a_{\mathrm{CMY}-2}$ gene to extended-spectrum cephalosporins. In the European clade, $b l a_{\mathrm{TEM}-1 \mathrm{~B} / 1 \mathrm{~B}}$-like was mainly found on IncI1 plasmids $(17 / 42)$ and, to a lesser extent, on IncX4 (7/42) and IncHI2 (3/42) plasmids. In strains from the Latin American clade, $b l a_{\text {СMY-2 }}$ was mainly found on IncI1/ST12 plasmids (25/33) (Figure 1). The sul2 gene, conferring resistance to sulphonamides, was frequently encountered in strains from Europe (48\%) and Latin America (14\%). In the European clade, sul2 was mainly found to co-localize with bla $a_{\text {TEM-1B } / 1 \mathrm{~B}}$-like on IncI1 plasmids (17/30), whereas in the Latin American clade, sul2 was found on ColRNAI-like plasmids (9/9). $\beta$-lactam and sulphonamide resistance genes in the Latin American clade were observed only in strains from Colombia (Figure 1). In addition, $>50 \%$ of strains from the European clade exhibited known DNA gyrase mutations conferring resistance to fluoroquinolones. The observed mutations were gyrA D87G (16/33), D87Y (10/33), and S83F (7/33) (Figure 1). Although no strains from the Latin American clade carried the chromosomal gyrA mutations, they did carry qnrB19-harboring plasmids in $96 \%$ of the cases. qnrB19 is known to confer reduced susceptibility to quinolones. This gene was co-localized with ColRNAI-like plasmids in strains from Latin America and in 1 strain from Europe from recent years (2015) (Figure 1).

\section{PCA of Accessory Genomes}

We observed a marked distinction for the accessory genes located on chromosome contigs between the European and Latin American clades (Figure 3). The separation in the PCA was associated with the presence of a prophage highly similar to the Salmonella phage SEN34 (National Center for Biotechnology Information reference sequence NC_028699.1) in strains from Latin America. This phage was also found in the genome of a Salmonella serovar Saintpaul strain submitted in Canada (GenBank accession no. 

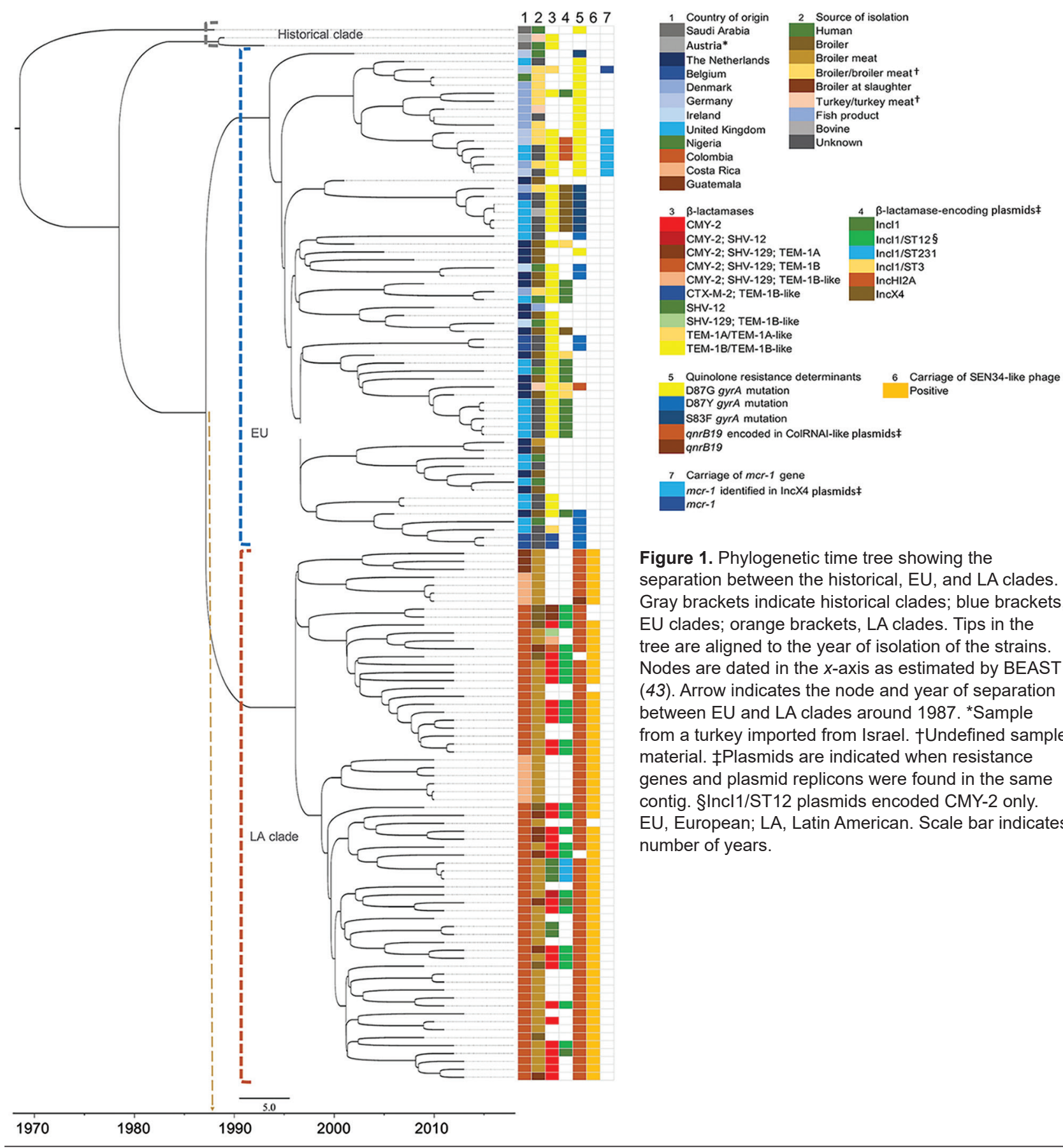

Figure 1. Phylogenetic time tree showing the separation between the historical, EU, and LA clades. Gray brackets indicate historical clades; blue brackets, EU clades; orange brackets, LA clades. Tips in the tree are aligned to the year of isolation of the strains. Nodes are dated in the $x$-axis as estimated by BEAST (43). Arrow indicates the node and year of separation between EU and LA clades around 1987. *Sample from a turkey imported from Israel. †Undefined sample material. $\ddagger$ Plasmids are indicated when resistance genes and plasmid replicons were found in the same contig. §Incl1/ST12 plasmids encoded CMY-2 only. EU, European; LA, Latin American. Scale bar indicates number of years.

CP022491.1). A few strains from Colombia closer to the cluster of strains from Europe in the PCA (UGBOG142, UGVIL373, and SSIII_4_C2) lacked the sequence of this phage. Plasmid composition was similar in strains from Europe and Latin America (Figure 4). The profiles of plasmid composition were characterized by the presence of IncI1 plasmids (cluster I), IncHI2 (cluster II), ColRNAI-like (cluster III), and combinations of IncI1 and IncHI2 plasmids (cluster
IV). Although the IncI1 plasmids had different pMLST sequence types, their content appears to be remarkably similar as they are in proximity in the PCA plot (cluster I). When exploring this further using only plasmid contigs $\geq 50 \mathrm{~kb}$ in strains from both European and Latin American clades, we observed that the IncI1 plasmid contigs clearly have near identical content (cluster I in Appendix 2 Figure 2). In strains with multiple plasmid contigs, in addition to the cluster of 


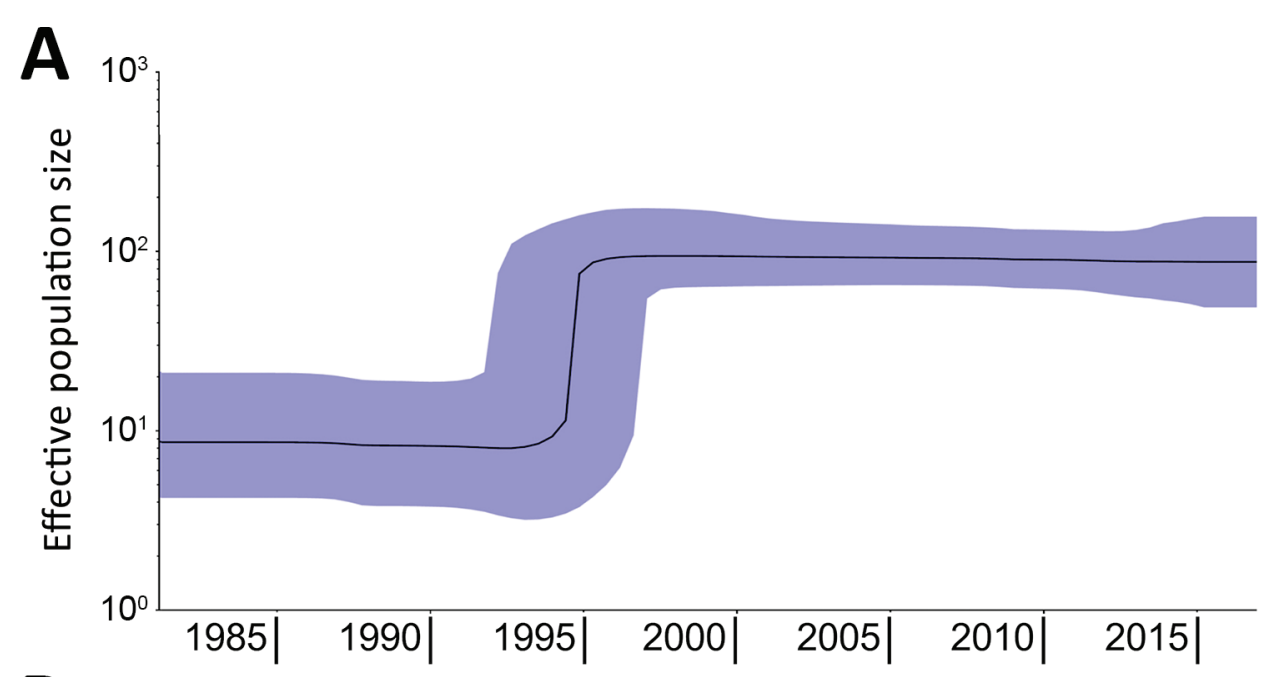

Figure 2. Bayesian skyline plots showing increase in effective population size of Salmonella enterica serovar Paratyphi B variant Java sequence type 28. Plots were made separately with strains originating from Europe $(A)$ or Latin America (B). Emergence in Europe occurred in $\approx 1995$ and in Latin America in $\approx 2005$. Black lines indicate estimates of the median population over time; purple shading indicates $95 \% \mathrm{Cls}$.

B

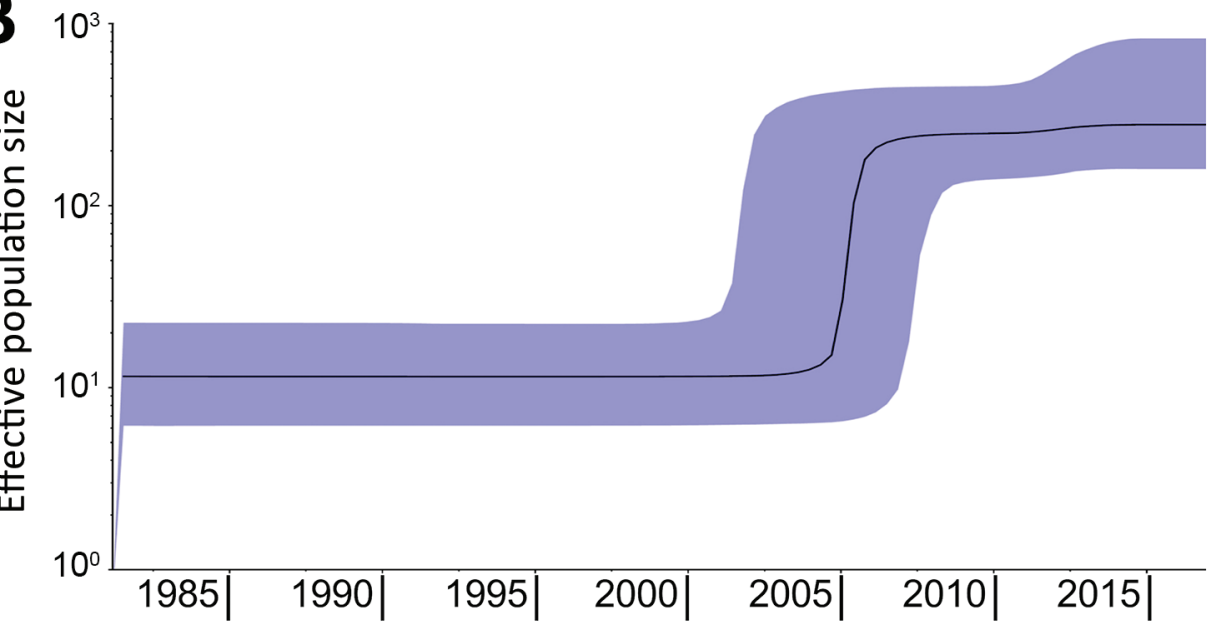

IncI1-like plasmids, IncHI2-like plasmid contigs were differentiated in cluster II (Appendix 2 Figure 2).

\section{Discussion}

We selected public genomes using EnteroBase and based on metadata availability and in silico serovar and MLST characterization. In a comparison between EnteroBase, ENA, and GenBank (Appendix 1 Table 2), we found 3,524 genomes associated to BioSamples (i.e., unique identifiers linking metadata and sequencing data) after querying EnteroBase for predicted serovar "Paratyphi B Var. Java." After querying "Salmonella AND Java," we found 1,245 in ENA and 1,299 in GenBank. This difference is mainly due to the large number of genomes submitted without serovar information $(1,935 / 3,524)$ rather than those labeled differently from Paratyphi B or variant Java (71 genomes). In this regard, in silico characterization in EnteroBase helped surpass absent, incorrect, or multiple serovar denominations accompanying genomes submitted to the ENA and GenBank databases.
On the basis of MLST, we found that poultry-associated variant Java ST28 (380 isolates), human-associated ST43 (767 isolates), and reptile-associated ST88 (319 isolates) were among the most abundant STs in EnteroBase. Most (143 isolates) ST28 genomes originated from poultry, and some (24 isolates) originated from humans, which could indicate transfer between these 2 sources. Because metadata were lacking on their year of isolation in EnteroBase (Appendix $1 \mathrm{Ta}-$ ble 2), some ST28 genomes were excluded from the phylogenetic time trees in this study. Nevertheless, phylogenetic analysis based on core genome MLST in EnteroBase (35) strongly supported the separate clustering of historical strains and those from Europe and Latin America (Appendix 2 Figure 3).

From the time-resolved phylogeny, we observed that strain IP_6155/87 collected in 1987 was ancestral to all the other strains analyzed. IP_6155/87 can be hypothesized to be the strain most closely related to the common ancestor of all analyzed strains, which according to the output from the time-resolved 


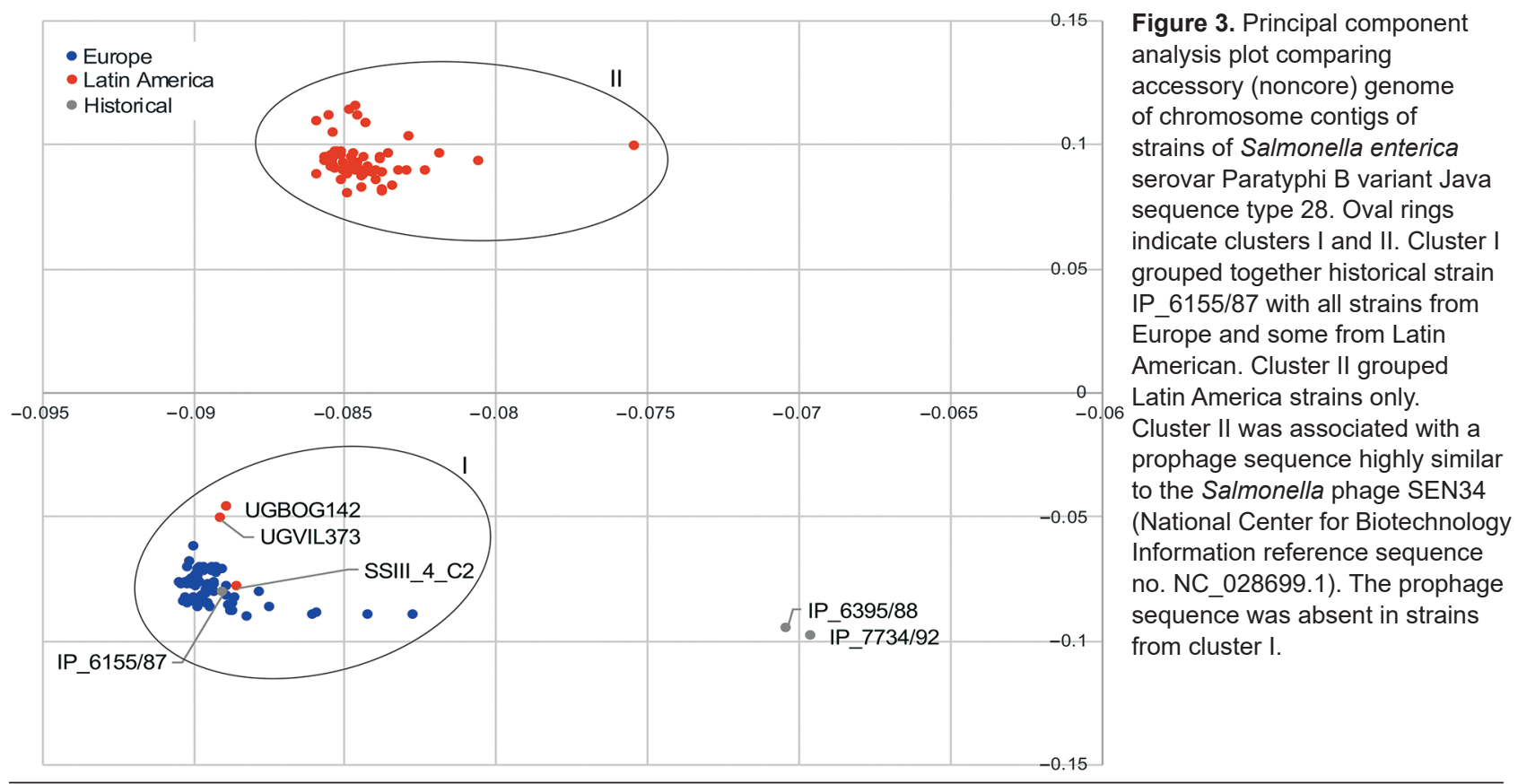

phylogeny circulated around 1970 (95\% CI 19621974). This hypothesis is supported by the high level of similarity between the contigs carrying the class 2 integron (with dfrA1-sat1-aadA1 [GenBank accession no. AB188271.1]) between historical strain IP_6155/87 and strains from Europe and Latin America. Common ancestry of IP_6155/87 was also reflected in the PCAs used to compare the accessory genome. At both chromosome and plasmid levels, the accessory genome of this strain was closely related to strains from Europe, Latin America, or both (Figures 3, 4; Appendix 2 Figure 2).
In comparison with previous estimations of mutation rates for Salmonella Typhimurium DT104 (3.4× $10^{-7}$ substitutions/site/year) (48), the molecular clock calibrated for Salmonella Paratyphi B var. Java ST28 in our study was estimated at $3.5 \times 10^{-7}$ substitutions/ site/year, corresponding to $1.7 \mathrm{SNP} /$ genome/year (95\% CI 1.44-2.0 SNP/genome/year). Our results indicate similar mutation rates for these $2 \mathrm{~S}$. enterica serovars with distinct ecologic niches.

Previously, genomic characterization of Salmonella Paratyphi B var. Java ST28 from Colombia suggested a different clade from the one observed

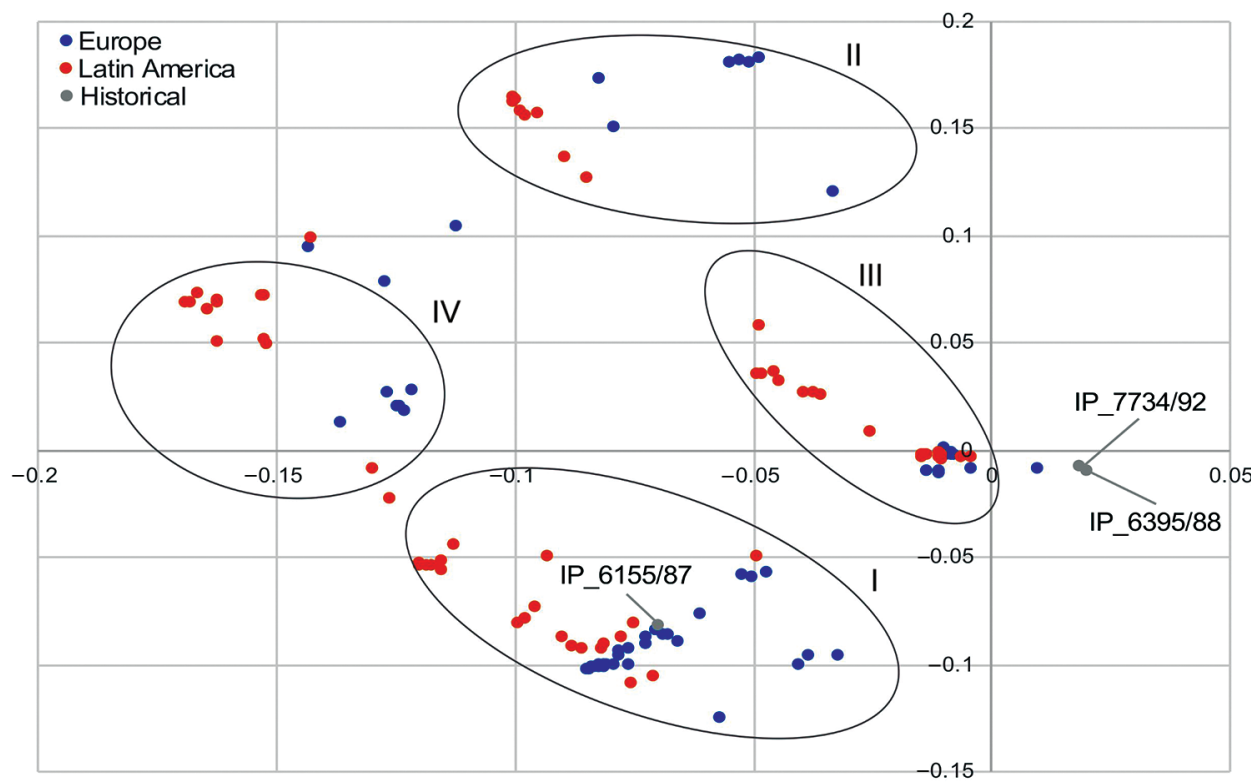

Figure 4. Principal component analysis plot comparing plasmid composition (all plasmid contigs) of strains of Salmonella enterica serovar Paratyphi B variant Java sequence type 28 . Oval rings indicate clusters I-IV. All clusters grouped strains from Europe and Latin America and were associated with Incl1 plasmids (cluster I), IncHI2 (cluster II), COLRNAI (cluster III), and combinations of Incl1 and IncHI2 plasmids (cluster IV). 
in Europe could be circulating in Latin America (31). We found a distinct clade of Salmonella Paratyphi B var. Java ST28 circulating in poultry from Costa Rica, Guatemala, and Colombia. In Colombia, introduction of foreign technologies for poultry breeding, housing, and processing occurred around 1960 (49). Importation of this particular S. enterica serovar was anticipated to have occurred around this time. Nevertheless, separation between the European and Latin American clades in our study was estimated with BEAST in the 1980s (Figure 1; Appendix 2 Figure 1). Furthermore, an increase in effective population size in Latin America was observed only in 2005 (95\% CI 2001-2007), 10 years after the known increase in Europe, reported in the literature (19) and observed with Bayesian skyline in our study (Figure 2). Driving factors that led to the separation of clusters could not be determined based on the availability of data for this study.

The separation between the Latin American and European clades comprised differences at both the core and noncore genome level. Differences in antimicrobial resistance gene content, plasmid replicons, and pMLSTs reflected the evolutionary separation of the 2 clades. Among these differences, DNA gyrase mutations conferring resistance to fluoroquinolones and $b l a_{\text {TEM-1B }}$-carrying IncI1 plasmids were characteristic in the European clade. In contrast, qnrB19carrying ColRNAI-like plasmids conferring reduced susceptibility to quinolones and $b l a_{\mathrm{CMY}-2}$-carrying IncI1/ST12 plasmids were found in Latin America. In both clades, resistance to $\beta$-lactams was mainly carried on IncI1 plasmids, with near-identical gene content (cluster I in Figure 4) but from different pMLST lineages. bla $a_{\text {TEM-1B }}$ in Europe was associated with IncI/ST3 in some strains and bla $a_{\mathrm{CMY}-2}$ with IncI1/ST12 in Latin America. It is remarkable that the genomic features confer resistance to $\beta$-lactam drugs and quinolones/fluoroquinolones in both European and Latin American clades and thus indicate parallel evolution of Salmonella Paratyphi B var. Java ST28 in both geographic regions. Acquisition of such antimicrobial resistance traits can be hypothesized to have occurred as a consequence of selection pressure posed by the use of $\beta$-lactams and fluoroquinolones in poultry production. As an example, and as previously suggested, the use of aminopenicillins in broiler production potentially could have selected for $b l a_{\mathrm{CMY}-2}$-carrying $E$. coli strains in the absence of the use of cephalosporins (50). Furthermore, the emergence of Salmonella Paratyphi B var. Java in Europe was associated with increased antimicrobial resistance and the use of fluoroquinolones, such as flumequine (19) or enrofloxacin, which could also explain the sharp increase in effective population size around 1995. For Latin America, such information is not available because data on the use of antimicrobial drugs in animals is not systematically collected in most countries in the region.

In conclusion, Salmonella Paratyphi B var. Java ST28 from poultry in Europe and Latin America form 2 different clades. The separation is estimated to have occurred during the 1980s (95\% CI 1978-1988). The years with sharp increase in effective population size were estimated as 1995 for Europe and 2005 for Latin America. Previous reports about the emergence of Salmonella Paratyphi B var. Java in poultry in Europe supports these findings for Europe $(6,19)$; no historical data are available for Latin America. In spite of their evolutionary divergence, the European and Latin American clades have independently acquired different antimicrobial resistance genes on similar plasmids. These genetic determinants confer resistance to $\beta$-lactam drugs and quinolones and thus indicate parallel evolution of Salmonella Paratyphi B var. Java ST28 in both regions.

\section{Acknowledgments}

We thank Birgitta Duim, Arjen Timmerman, Alice Wegener, and Sylvie Issenhuth-Jeanjean for assistance in obtaining the WGS of strains. We also thank Johan F. Bernal, Maribel León, Alejandra Arevalo, María F. Valencia, and Viviana Clavijo for their assistance during the collection and characterization of Salmonella strains from Colombia. We thank Walid Alali and Enrique PerezGutierrez for their contribution to the projects where the prevalence of $S$. enterica in poultry from Colombia and Guatemala was determined. We thank Jaap Obdam for providing historical information on Salmonella serovars in poultry production in the Netherlands. We thank Alison E. Mather for her constructive revision of the manuscript. We acknowledge the World Health Organization Advisory Group on Integrated Surveillance of Antimicrobial Resistance for facilitating the exchange of researchers and knowledge between research groups.

This study was financed by internal funding of Utrecht University, the Netherlands.

\section{About the Author}

Dr. Castellanos is a postdoctoral research scientist at the Quadram Institute Bioscience in Norwich, UK. His primary research interests are the evolution of bacteria and plasmids and the reduction of antimicrobial resistance in humans, animals, and food. 


\section{References}

1. De Moor CE. Salmonella infection in Batavia (Java), with particular reference to the diagnosis of the type of infection [in German]. Mededeelingen van den Dienst der Volksgzondheid in Nederlandsch-Indië. 1935;24:98-118.

2. Kristensen M, Kauffmann F. Bakteriologische und klinische Erfahrungen über Infektionen mit d-weinsäurevergärenden Paratyphus B-Bacillen. Z Hyg Infektionskr. 1937;120:149-54. https://doi.org/10.1007/BF02178014

3. Kauffmann F. Differential diagnosis and pathogenicity of Salmonella Java and Salmonella Paratyphi B [in German]. Z Hyg Infektionskr. 1955;141:546-50. https:/ / doi.org/10.1007/BF02156850

4. Barker RM, Kearney GM, Nicholson P, Blair AL, Porter RC, Crichton PB. Types of Salmonella Paratyphi B and their phylogenetic significance. J Med Microbiol. 1988;26:285-93. https:/ / doi.org/10.1099/00222615-26-4-285

5. Miko A, Guerra B, Schroeter A, Dorn C, Helmuth R. Molecular characterization of multiresistant $d$-tartrate-positive Salmonella enterica serovar Paratyphi B isolates. J Clin Microbiol. 2002;40:3184-91. https://doi.org/10.1128/ JCM.40.9.3184-3191.2002

6. Brown DJ, Mather H, Browning LM, Coia JE. Investigation of human infections with Salmonella enterica serovar Java in Scotland and possible association with imported poultry. Euro Surveill. 2003;8:35-40. https:/ / doi.org/10.2807/ esm.08.02.00399-en

7. Weill FX, Fabre L, Grandry B, Grimont PAD, Casin I. Multipleantibiotic resistance in Salmonella enterica serotype Paratyphi B isolates collected in France between 2000 and 2003 is due mainly to strains harboring Salmonella genomic islands 1, 1-B, and 1-C. Antimicrob Agents Chemother. 2005;49:2793-801. https://doi.org/10.1128/AAC.49.7.2793-2801.2005

8. Threlfall J, Levent B, Hopkins KL, de Pinna E, Ward LR, Brown DJ. Multidrug-resistant Salmonella Java. Emerg Infect Dis. 2005;11:170-1. https://doi.org/10.3201/eid1101.031092

9. Denny J, Threlfall J, Takkinen J, Lofdahl S, Westrell T, Varela C, et al. Multinational Salmonella Paratyphi B variant Java (Salmonella Java) outbreak, August-December 2007. Euro Surveill. 2007;12:E071220.2.

10. Doublet B, Praud K, Nguyen-Ho-Bao T, Argudín MA Bertrand S, Butaye P, et al. Extended-spectrum $\beta$-lactamaseand AmpC $\beta$-lactamase-producing D-tartrate-positive Salmonella enterica serovar Paratyphi B from broilers and human patients in Belgium, 2008-10. J Antimicrob Chemother. 2014;69:1257-64. https://doi.org/10.1093/jac/ dkt504

11. Meunier D, Boyd D, Mulvey MR, Baucheron S, Mammina C, Nastasi A, et al. Salmonella enterica serotype Typhimurium DT 104 antibiotic resistance genomic island I in serotype Paratyphi B. Emerg Infect Dis. 2002;8:430-3. https://doi.org/ 10.3201/eid0804.010213

12. Mulvey MR, Boyd D, Cloeckaert A, Ahmed R, Ng LK; Provincial Public Health Laboratories. Emergence of multidrug-resistant Salmonella Paratyphi B dT+, Canada. Emerg Infect Dis. 2004;10:1307-10. https:/ / doi.org/10.3201/ eid1007.030862

13. Gaulin C, Vincent C, Ismaiil J. Sporadic infections of Salmonella Paratyphi B, var. Java associated with fish tanks. Can J Public Health. 2005;96:471-4. https:/ / doi.org/10.1007/ BF03405194

14. Hassan R, Tecle S, Adcock B, Kellis M, Weiss J, Saupe A, et al. Multistate outbreak of Salmonella Paratyphi B variant $\mathrm{L}(+)$ tartrate $(+)$ and Salmonella Weltevreden infections linked to imported frozen raw tuna: USA, March-July 2015. Epidemiol Infect. 2018;146:1461-7. https:/ / doi.org/10.1017/ S0950268818001462
15. Heiman Marshall KE, Booth H, Harrang J, Lamba K, Folley A, Ching-Lee M, et al. New product, old problem(s): multistate outbreak of Salmonella Paratyphi B variant L(+) tartrate $(+)$ infections linked to raw sprouted nut butters, October 2015. Epidemiol Infect. 2019;8:1-6.

16. Levings RS, Lightfoot D, Hall RM, Djordjevic SP. Aquariums as reservoirs for multidrug-resistant Salmonella Paratyphi B. Emerg Infect Dis. 2006;12:507-10. https:/ / doi.org/10.3201/ eid1203.051085

17. Djordjevic SP, Cain AK, Evershed NJ, Falconer L, Levings RS, Lightfoot D, et al. Emergence and evolution of multiply antibiotic-resistant Salmonella enterica serovar Paratyphi B D-tartrate-utilizing strains containing SGI1. Antimicrob Agents Chemother. 2009;53:2319-26. https://doi.org/10.1128/AAC.01532-08

18. Toboldt A, Tietze E, Helmuth R, Fruth A, Junker E, Malorny B. Human infections attributable to the D-tartratefermenting variant of Salmonella enterica serovar Paratyphi $\mathrm{B}$ in Germany originate in reptiles and, on rare occasions, poultry. Appl Environ Microbiol. 2012;78:7347-57. https://doi.org/10.1128/AEM.01732-12

19. van Pelt $W$, van der Zee $H$, Wannet $W J$, van de Giessen AW, Mevius DJ, Bolder NM. Explosive increase of Salmonella Java in poultry in the Netherlands: consequences for public health. Euro Surveill. 2003;8:31-5. https:// doi.org/10.2807/ esm.08.02.00398-en

20. Centrum voor Onderzoek in Diergeneeskunde en Agrochemie. Centre d'Etude et des Recherches Vétérinaires et Agrochimiques (CODA-CERVA). Salmonella serotypes analysed at the CODA-

CERVA in 2013. Technical Report. Federal Public Service Health, Food Chain Security and Environment:Brussels: 2014.

21. Achtman M, Wain J, Weill FX, Nair S, Zhou Z, Sangal V, et al.; S. Enterica MLST Study Group. Multilocus sequence typing as a replacement for serotyping in Salmonella enterica. PLoS Pathog. 2012;8:e1002776. https://doi.org/10.1371/ journal.ppat.1002776

22. Connor TR, Owen SV, Langridge G, Connell S, Nair S, Reuter S, et al. What's in a name? Species-wide wholegenome sequencing resolves invasive and noninvasive lineages of Salmonella enterica serotype Paratyphi B. MBio. 2016;7:1-9. https://doi.org/10.1128/mBio.00527-16

23. Doumith M, Godbole G, Ashton P, Larkin L, Dallman T, Day M, et al. Detection of the plasmid-mediated $m c r-1$ gene conferring colistin resistance in human and food isolates of Salmonella enterica and Escherichia coli in England and Wales. J Antimicrob Chemother. 2016;71:2300-5. https://doi.org/10.1093/jac/dkw093

24. Borowiak M, Fischer J, Hammerl JA, Hendriksen RS, Szabo I, Malorny B. Identification of a novel transposon-associated phosphoethanolamine transferase gene, $m c r-5$, conferring colistin resistance in $d$-tartrate fermenting Salmonella enterica subsp. enterica serovar Paratyphi B. J Antimicrob Chemother. 2017;72:3317-24. https:// doi.org/10.1093/jac/dkx327

25. Castellanos LR, van der Graaf-van Bloois L, Donado-Godoy P, Mevius DJ, Wagenaar JA, Hordijk J, et al. Phylogenomic investigation of IncI1-IY plasmids harboring $b l a_{\mathrm{CMY}-2}$ and $b l a_{\mathrm{SHV}-12}$ in Salmonella enterica and Escherichia coli in multiple countries. Antimicrob Agents Chemother. 2019;63:e02546-18. https://doi.org/10.1128/AAC.02546-18

26. Donado-Godoy P, Gardner I, Byrne BA, Leon M, Perez-Gutierrez E, Ovalle MV, et al. Prevalence, risk factors, and antimicrobial resistance profiles of Salmonella from commercial broiler farms in two important poultryproducing regions of Colombia. J Food Prot. 2012;75:874-83. https://doi.org/10.4315/0362-028X.JFP-11-458 
27. Donado-Godoy P, Clavijo V, León M, Arevalo A, Castellanos R, Bernal J, et al. Counts, serovars, and antimicrobial resistance phenotypes of Salmonella on raw chicken meat at retail in Colombia. J Food Prot. 2014;77:22735. https://doi.org/10.4315/0362-028X.JFP-13-276

28. Donado-Godoy P, Byrne BA, León M, Castellanos R, Vanegas C, Coral A, et al. Prevalence, resistance patterns, and risk factors for antimicrobial resistance in bacteria from retail chicken meat in Colombia. J Food Prot. 2015;78:751-9. https:/ / doi.org/10.4315/0362-028X.JFP-14-349

29. Jarquin C, Alvarez D, Morales O, Morales AJ, López B, Donado $\mathrm{P}$, et al. Salmonella on raw poultry in retail markets in Guatemala: levels, antibiotic susceptibility, and serovar distribution. J Food Prot. 2015;78:1642-50. https:/ / doi.org/10.4315/0362-028X.JFP-15-117

30. Boscán-Duque LA, Arzálluz-Fisher AM, Ugarte C, Sánchez D, Wittum TE, Hoet AE. Reduced susceptibility to quinolones among Salmonella serotypes isolated from poultry at slaughter in Venezuela. J Food Prot. 2007;70:2030-5. https://doi.org/10.4315/0362-028X-70.9.2030

31. Castellanos LR, van der Graaf-van Bloois L, Donado-Godoy P, León M, Clavijo V, Arévalo A, et al. Genomic characterization of extended-spectrum cephalosporin-resistant Salmonella enterica in the Colombian poultry chain. Front Microbiol 2018;9:2431. https://doi.org/10.3389/fmicb.2018.02431

32. Donado-Godoy P, Castellanos R, León M, Arevalo A, Clavijo V, Bernal J, et al. The establishment of the Colombian Integrated Program for Antimicrobial Resistance Surveillance (COIPARS): a pilot project on poultry farms, slaughterhouses and retail market. Zoonoses Public Health. 2015;62(Suppl 1):58-69. https:// doi.org/10.1111/zph.12192

33. Ministerio de Agricultura y Ganadería, Instituto Costarricense de Investigación y Enseñanza en Nutrición y Salud, Ministerio de Salud. Prevalence of Salmonella spp. in meat and chicken products. Costa Rica, September-November 2009 [in Spanish]. Technical Report. Ministry of Agriculture and Livestock, Costa Rican Institute for Research and Training in Nutrition and Health and Ministry of Health. San Jose (Costa Rica); 2011.

34. Veldman K, Mevius DJ, Wit B, van Pelt W, Heederik D. MARAN. Monitoring of antimicrobial resistance and antibiotic usage in animals in the Netherlands in 2016. 2017 [cited 2019 Mar 7]. https:/ / www.wur.nl/upload_mm/9/b/4/fe79278b9361-4912-8cba-03ce17fc086b_Maran\%20report\%202017.pdf

35. Alikhan NF, Zhou Z, Sergeant MJ, Achtman M. A genomic overview of the population structure of Salmonella. PLoS Genet. 2018;14:e1007261. https://doi.org/10.1371/journal. pgen.1007261

36. Kuijpers LMF, Phe T, Veng $\mathrm{CH}$, Lim $\mathrm{K}$, Ieng S, Kham C, et al. The clinical and microbiological characteristics of enteric fever in Cambodia, 2008-2015. PLoS Negl Trop Dis. 2017;11:e005964. https://doi.org/10.1371/journal. pntd.0005964

37 Bankevich A, Nurk S, Antipov D, Gurevich AA, Dvorkin M, Kulikov AS, et al. SPAdes: a new genome assembly algorithm and its applications to single-cell sequencing. J Comput Biol. 2012;19:455-77. https:// doi.org/10.1089/cmb.2012.0021

38. Zankari E, Hasman H, Cosentino S, Vestergaard M, Rasmussen $\mathrm{S}$, Lund $\mathrm{O}$, et al. Identification of acquired antimicrobial resistance genes. J Antimicrob Chemother. 2012;67:2640-4. https://doi.org/10.1093/jac/dks261

39. Carattoli A, Zankari E, García-Fernández A, Voldby Larsen M, Lund $\mathrm{O}$, Villa L, et al. In silico detection and typing of plasmids using PlasmidFinder and plasmid multilocus sequence typing. Antimicrob Agents Chemother. 2014;58:3895-903. https:// doi.org/10.1128/AAC.02412-14

40. Larsen MV, Cosentino S, Rasmussen S, Friis C, Hasman H, Marvig RL, et al. Multilocus sequence typing of totalgenome-sequenced bacteria. J Clin Microbiol. 2012;50:135561. https:/ / doi.org/10.1128/JCM.06094-11

41. Treangen TJ, Ondov BD, Koren S, Phillippy AM. The Harvest suite for rapid core-genome alignment and visualization of thousands of intraspecific microbial genomes. Genome Biol. 2014;15:524. https:// doi.org/10.1186/s13059-014-0524-x

42. Croucher NJ, Page AJ, Connor TR, Delaney AJ, Keane JA, Bentley SD, et al. Rapid phylogenetic analysis of large samples of recombinant bacterial whole genome sequences using Gubbins. Nucleic Acids Res. 2015;43:e15. https://doi.org/10.1093/nar/gku1196

43. Drummond AJ, Rambaut A. BEAST: Bayesian evolutionary analysis by sampling trees. BMC Evol Biol. 2007;7:214. https://doi.org/10.1186/1471-2148-7-214

44. Seemann T. Prokka: rapid prokaryotic genome annotation. Bioinformatics. 2014;30:2068-9. https:/ / doi.org/10.1093/ bioinformatics/btu153

45. Page AJ, Cummins CA, Hunt M, Wong VK, Reuter S, Holden MTG, et al. Roary: rapid large-scale prokaryote pan genome analysis. Bioinformatics. 2015;31:3691-3. https://doi.org/10.1093/bioinformatics/btv421

46. Parks DH, Imelfort $\mathrm{M}$, Skennerton CT, Hugenholtz P, Tyson GW. CheckM: assessing the quality of microbial genomes recovered from isolates, single cells, and metagenomes. Genome Res. 2015;25:1043-55. https://doi.org/10.1101/gr.186072.114

47. Arndt D, Grant JR, Marcu A, Sajed T, Pon A, Liang Y, et al. PHASTER: a better, faster version of the PHAST phage search tool. Nucleic Acids Res. 2016;44(W1):W16-21. https://doi.org/10.1093/nar/gkw387

48. Mather AE, Reid SWJ, Maskell DJ, Parkhill J, Fookes MC, Harris SR, et al. Distinguishable epidemics of multidrugresistant Salmonella Typhimurium DT104 in different hosts. Science. 2013;341:1514-7. https://doi.org/10.1126/science. 1240578

49. Molina LF. Poultry farming in Colombia [in Spanish]. Federación Nacional de Avicultores de Colombia (Fenavi)-Fondo Nacional Avícola (Fonav): Bogotá (Colombia); 2002.

50. Agersø $\mathrm{Y}$, Jensen JD, Hasman H, Pedersen K. Spread of extended spectrum cephalosporinase-producing Escherichia coli clones and plasmids from parent animals to broilers and to broiler meat in a production without use of cephalosporins. Foodborne Pathog Dis. 2014;11:740-6. https:/ / doi.org/10.1089/ fpd.2014.1742

Address for correspondence: Aldert L. Zomer, Department of Infectious Diseases and Immunology, Faculty of Veterinary Medicine, Utrecht University, Yalelaan 1, 3584CL, Utrecht, the Netherlands; email: a.1.zomer@uu.nl 